\title{
Draft-Minutes of the 2nd General Assembly of the European Association for the Study of Diabetes, held in the Institute of Anatomy, University of Aarhus Denmark, on Thursday, 7th July 1966, at 18:10
}

\author{
Present: Dr. F.G. Young (President) \\ Dr. K. LU்NDBAEK (Vice-president) \\ Dr. E. Martin (Vice-president) \\ Dr. E. F. Pfenffer (Treasurer) \\ Dr. A. E. Renold (Secretary) \\ and 108 members of the Association.
}

\section{Minutes}

The President reported that the Minutes of the First General Assembly had been printed in the June 1966 issue of Diabetologia $(\mathbf{2}, 1,72-74)$. There were two amendments :

a) Minute 3: In both the English and French versions of the Minutes, the number of members of the Editorial Board had inadvertently been printed as 17 instead of 26 or more.

b) Minute 7: In the English version only, the names of the elected Honorary Auditors had been omitted.

The corrections to the Minutes having been made, the Minutes of the First Genera] Assembly were taken as read, confirmed as a correct record, and signed by the President.

\section{Report of the President}

The President said that in his remarks at the opening of the Association meeting, he had expressed the thanks of the Association to Dr. K. LUNDBAEK and his colleagues for their organisation of the meeting, and had anticipated its success. He was sure that his expectations had been fully justified.

The Secretary would be reporting on the organisational aspect of the meeting but the President emphasised the value of the Abstracts, printed in at least two languages and made available through the generosity of Novo Laboratories; and the facilities for informal discussions, which were most helpful.

The President went on to express the Association's thanks for the devoted service given by the Officers of the Association and said that the Statutes adopted at the first General Assembly were working well in practice, although the Secretary would be proposing a temporary suspensions of Article 3, Section 16 in order to avoid the retirement of all members of the existing Executive Committee simultaneously.

Referring to the Minkowski Award, the President reported that the regulations for the Award had been adopted by the Council. The standard of nominees had been extremely high and he was sure that the Association could look forward to a long series of excellent Minkowski Lectures, such as had been delivered by the first recipient, Dr. P.J. RANDLE.

The Award Committee consisted of four members appointed by the Council, one of whom retired each year, and one nominee of the sponsors, Farbwerke Hoechst A.G. Dr. H. MASKE had tendered his resignation from the Award Committee and the Council had appointed Dr. A. Loubatrìres in his place. Dr. voN WASIELEWSKI had been nominated by Farbwerke Hoechst A. G. and had been appointed to the Committee.

The Council had agreed that there should be no meeting of the European Association in the years in which the International Diabetes Federation met in Europe but it had been agreed with the Federation that the Minkowski Prize could be presented during the Congress of the Federation, and that the Minkowski Lecture could be deliv. ered as part of the proceedings of the Congress.
The President went on to say that the Council had agreed to encourage the holding of an Exhibition in conjunction with the meetings of the Association and ended by saying that the Council were of the opinion that future meetings of the Association should be held preferably in relatively small University Towns. It had been suggested that future meetings might be held on fixed dates in each year and the President asked for an expression of opinion as to whether a fixed date was desirable.

By a show of hands, the General Assembly indicated by a large majority that it was in favour of meetings being held annually in the early part of July, although they recognised that this might not always be practical.

\section{Report of the Treasurer}

The President invited Dr. E.F. PFEIFFER to give his report.

The Treasurer began by presenting the financial position of the Association as follows:

Credits: 13. 10.64 - 30.6.66 S.fr. 56597.95

Debits: $13.10 .64-30.6 .66$ S.fr. 19639.95

Balance as $\quad$ at 30.6.66 S.fr. 36958.00

The great majority of the Credits had been received from 12 Supporting Members of the Association and he was happy to report that 2 further Supporting Members had been enrolled during the current Meeting.

Referring to the expenditure, Dr.PFETFFER emphasised the excellent work of the Secretariat in Geneva whose costs had been kept remarkably low: but as the Associaton grew, so would the expenses, and he estimated that the Secretariat costs for $1966 / 67$ would be S.fr. 15000.annually.

The other major costs under expenditure arose through Diabetologia and the Treasurer reported that the engagement of a permanent secretary for the considerable work involved in this publication was now essential, in addition to the part-time secretary already in office. The publishers, Springer-Verlag, had generously agreed to meet half the cost of both secretaries. Other payments were in respect of translations, and the subvention paid to the publishers in respect of members of the Association who received Diabetologia at the special reduced rate. Some savings could be made in the cost of production if authors would write their own translations of summaries, and it was hoped that this could be done.

Including the Secretariat, and the cost involved in the publication of Diabetologia, the Treasurer estimated that an annual budget of S.fr. 33000 . - would be required for $1966 / 67$.

Dr. Pheifferer continued by mentioning some of the proposals under discussion to reduce the expenses of the Association. It was proposed to introduce an Admission Fee at future meetings of the Association, which would cover both the cost of the Abstracts and of certain meals. It was also intended to encourage the holding of an Exhibition in conjunction with the meetings.

The Treasurer asked all mombers to try and encourage the enrolment of more Supporting Members.

On the collections of membership dues, Dr. PFEIFFER expressed the hope that national associations might be able to play their part in arranging to collect and transfer in bulk the small sums involved, although it was recog. nised that membership of the European Association was an individual matter, and that not all members of national associations might wish to become members of the European Associations. 
Dr. PFeIfFer said that it was his aim to raise S.fr. $50000 .-$ annually. Much of the Editorial work for Diabetologia was at present carried out without charge, but this could not be expected to continue indefinitely. In addition, it was his hope that the Association would be able to give financial support to young workers to enable them to attend the meetings and to present papers.

The Treasurer closed his report by saying that in the absence from Aarhus of Dr. M. Coppo, one of two elected Honorary Auditors, Dr. J. Mirouze had been invited to act as joint Honorary Auditor with Dr. P. RAMBERT. Dr. P. RAMBert and Dr. J. Mirouze were duly proposed as Honorary Auditors for 1966/1967 and elected by acclamation:

In reply to a question, the Treasurer stated that approximately S.fr. 6300. - was received from membership dues.

\section{Report of the honorary auditors}

The President invited Dr. P. Rambert to give his report.

Dr. P. Rambert, on behalf of Dr. J. Mrrouze and himself, advised the General Assembly that the Accounts had been kept perfectly, and he was happy to recommend to the General Assembly that the Treasurer be discharged from all responsibility for the Accounts up to 31st December, 1965. The General Assembly unanimously approved the proposal.

The President expressed the thanks of the Association to Dr. RaviBert and Dr. Mrrouze for their work, and went on to voice the Association's appreciation of the able and lucid report of the Treasurer, and of all his hard work.

\section{Report of the secretary}

The President invited Dr. A.E. Renoxd to give his report.

The Secretary began by saying that there were $905 \mathrm{Ac}$ tive Members of the Association, of whom approximately 500 had paid their membership dues. Reminders would be sent in due course to those who had not yet paid. Of the 905 members, only 225 were subscribers to Diabetologia, and he appealed to all members to support this publication.

Dr. Renold then reported that the names of the members of the Council, of the Honorary Members, and of the Supporting Members would be printed in future issues of Diabetologia.

Turning to the subject of the meetings, the Secretary emphasised the benefits received through the generous support of a number of firms. He was sure that members appreciated that it had not been possible to allow time for the presentation of all the 180 papers of which the Abstracts had been printed, and he asked for the com. ments of members on the form adopted at the Aarhus meeting, whereby papers had been limited to 15 minutes presentation, followed by 10 minutes of discussion. It was felt that this was preferable to having parallel sessions. In addition, the interpolation of 2 minute interventions with no more than 2 slides had worked well.

On a show of hands, the majority of members present favoured the continuation of this policy for future meetings.

Finally, the Secretary reported that Article 3, Section 16 of the Statutes provided for the retirement of all members of the Council after three years service. It was felt that the retirement of the initial Executive Committee (the President, two Vice-Presidents, the Treasurer and the Secretary) at the same time would be most inconvenient, and the Council therefore proposed that the provisions of this Section of the Statutes should be temporarily suspended to allow for the retirement of the first Executive Committee over a maximum period of five years, as from 1965 .
The General Assembly approved this proposal, and it was left to the Council to decide the order of retirement.

There being no question on the Secretary's report, the President thanked Dr. RENoLd most warmly for all his work as Secretary for which the Association was greatly indebted.

\section{Report of the editor-in-chief of Diabetologia}

The President invited Dr. K. OBERdissE, Editor-inchief of Diabetologia to give his report. Dr. OBERDISSE said that the first number of the publication had been issued in August 1965. Thanks to the quality of the contributed papers and to the intensive propaganda of the publishers, Springer-Verlag, the number of subscribers was now approximately 1000 .

There had been a steady flow of manuscripts, but the quality varied, and some manuscripts had to be rejected. The use of three languages had caused some major difficulties, but it was the aim of the Editorial Board to ensure regular production. With this in mind, the Board hoped that in future all authors would forward their manuscripts in triplicate to avoid the necessity of having them photo-copied; in addition, the summaries of the papers should be submitted in all three languages. The Editorial Board was willing to assist with the revision of translations but could not undertake to do the translations themselves.

It was hoped to standardise the use of abbreviations, and a small committeo had been established to make recommendations in this respect.

Two members of the Advisory Board had died, namely Dr. G Mohnike and Dr. R.F. OGILvie. In their place, Drs. G. Peters (Lausanne) and G. Seifent (Hamburg) had been appointed, and Drs. T. Steen-Olsen (Aarhus) and Dr. A. Hasserblate (Göttingen) had been added to the Board.

Dr. OBERDISSE emphasised that authors were themselves responsible for the checking of the galley proofs, and asked that more care be exercised in doing this, to help reduce the work of the Editorial Board, which was responsible for checking the page proofs.

Finally, the Editor-in-chief paid tribute to the help and advice he had received from the members of the Editorial and Advisory Boards and from the Officers of the Association. He also expressed his thanks to Dr. D.A.B. Young, Dr. Loubatik̀res, Dr. Daweike and Dr. Liebermeister for their help with the translations.

There being no questions, the President thanked Dr. OBERDISSE for all that he had done to ensure the fruitful start and continuation of the publication, and asked him to convey to his colleagues the Association's appreciation of their help.

\section{\%. Nominations and election of new council members}

The President reminded the General Assembly that at its first meeting, the Council had been charged with the responsibility for arranging the order of retirement of the members of the Council. This had been done by a draw, and the retiring members for 1966 were Drs. Alivisatos, Bartellemener, Chatn, Dérot and Pompen. The retiring members for 1967 would be Drs. JersiLD, LUFr, MASkE, RoDRIGUEz-MinoN and SignoRELLI. help.

The President thanked the retiring members for their

At the first General Assembly, three members, Drs. P.A. Bastexie, T.R. Fraser, and A. Loubatjères had been elected to the Council for the period 1966 - 1969 . There were therefore two vacancies, and the Council had pleasure in nominating Dr. A. CzYzYK (Warsaw) and Dr. I. Pavec (Bucarest) to fill these two places. There being no other nominations, Drs. Czyzyk and Paver were elected by acclamation. 


\section{Next meeting}

The President reported that the 3rd General Assembly would be held in Stockholm, Sweden, during the 6 th Congress of the.International Diabetes Federation from 30th July to 4th August 1967, at an hour and place to be announced.

\section{Any other business}

a) Membership list: In reply to a question, the Secre-

tary said that it was the intention of the Secretariat to produce a printed list of members of the Association within the next year.

There being no other business to discuss, the meeting ended.

Dr. F.G. YounG

President
Dr. A.E. RENoLD Secretary

\section{Procès-Verbal provisoire de la 2ème Assemblée Générale de l'Association Européenne pour l'Étude du Diabète, tenue à l'Institut d'Anatomie, Université d'Aarhus Danemark, le jeudi 7 juillet 1966, à $18 \mathrm{~h} .10$.}

\author{
Présents: Dr. F.G. Young (Président) \\ Dr. K. LundBaek (Vice-Président) \\ Dr. E. Martin (Vice-Président) \\ Dr. E.F. PFEIFFER (Trésorier) \\ Dr. A.E. Renold (Secrétaire) \\ et 108 membres de l'Association
}

\section{Procès-verbal}

Le Président annonce que le procès-verbal de la 1ère Assemblée générale a paru dans le numéro de juin 1966 de Diabetologia $(2,1,72-74)$. Deux modifications doivent lui être apportées:

a) 3: Les deux versions - anglaise et française - du procès-verbal mentionnent le nombre des membres du Comité de Rédaction de Diabetologia, comme étant de 17 au lieu de 26 ou plus.

b) 7: Dans la version anglaise seulement, le nom des vérificateur's des comptes a été omis.

Ainsi corrigé, le procès-verbal de la 1ère Assemblée générale est accepté et signé par le Président.

\section{Rapport du Président}

Le Président rappelle qu'à la séance d'ouverture, il avait exprimé la reconnaissance de l'Association au Dr. LUNDBAFK et à ses collaborateurs pour leur organisation, et escompté son succès. Il est sûr maintenant que tous les participants trouvent ces espoirs justifiés.

Il incombera au Secrétaire de faire rapport sur l'organisation de la réunion, mais le Président fait ressortir la valeur des résumés, imprimés en deux langues au moins, les traductions étant dues à la générosité des Laboratoires Novo. Il souligne également les dispositions prises pour qu'entre les présentations, les participants puissent aisément se rencontrer pour d'utiles discussions.

Le Président, au nom de l'Association, remercie ensuite les membres du Comité de leur concours. Il précise que les Statuts adoptés lors de la 1ère Assemblée générale sem. blent satisfaisants, en règle générale, mais que le Secrétaire proposera une suspension temporaire de l'article 16 pour éviter que l'actuel Comité exécutif ne se retire en bloc.

Quant au Prix Minkowski, le Président annonce que son règlement a été accepté par le Comité de Direction. Le niveau scientifique des candidats proposés a été extrêmement élevé, ce qui laisse prévoir une longue série d'excellentes conférences Minkowski, semblables à celle du premier lauréat, le Dr. P.J. RANDLE.

La Commission du Prix est formée de 4 membres nommés par le Comité de Direction (l'un deux se retirant annuellement) et d'un représentant proposé par Farbwerke Hoechst AG; le Dr. MASkr ayant présenté sa démission, le Comité de Direction a désigné le Dr. LoubaTIÈRES pour le remplacer. Le Dr. vON WASIELEWSKI ayant été proposé par Farbwerke Hoechst, le Comité de Direction l'a nommé membre de la Commission du Prix.
Le Comité de Direction a décidé qu'il n'y aurait pas de réunion de l'Association Européenne l'année où le Congrès de l'International Diabetes Federation se tiendrait en Europe, mais, en accord avec celle-ci, le Prix Minkowski sera attribué au cours de son congrès et la conférence du lauréat sera inscrite au programme.

Le Président annonce que le Comité de Direction a approuvé qu'une exposition accompagne les réunions annuelles de l'Association. Le Comité suggère que les réunions annuelles aient lieu dans des villes universitaires de moyenne importance, aussi souvent que possible. Il a été suggéré qu'une date fixe soit choisie et le Président demande à l'Assemblée d'exprimer son opinion à ce sujet. Par vote à main levée, une grande majorité se montre favorable à une date du début de juillet, en reconnaissant toutefois qu'il ne serait pas toujours possible de s'en tenir à cette date.

\section{Rapport du Trésorier}

Le Président invite le Dr. E. PfFiffFer à présenter son rapport. Le Trésorier définit la situation financière de I'Association de la manière suivante:

$$
\begin{aligned}
& \text { Crédits: 13. 10.64 - 30.6.66 S.fr. } 56597.95 \\
& \text { Débits: 13.10.64-30.6.66 S.fr. } 19639.95 \\
& \text { Bilan au 30. juin, } 1966 \quad \text { S.fr. } 36958.00
\end{aligned}
$$

La plupart des actifs sont dûs aux 12 membres bienfaiteurs, et le Trésorier est heureux d'annoncer que deux autres membres bienfaiteurs ont promis leur appui au. cours de cette réunion.

Quant aux frais généraux, le Dr. Preifrer souligne l'ex. cellent travail du secrétariat de Genève qui fonctionne très économiquement; mais l'Association se développant, les dépenses suivront une même courbe ascendante et il estime que les frais de secrétariat s'élèveront, en 1966/67, à $15000 \mathrm{fr}$. suisses par an.

C'est Diabetologia qui représente le second poste important des dépenses. Le Trésorier annonce qu'il est indis. pensable maintenant d'avoir une secrétaire permanente pour faire face au travail considérable entraîné par la publication, en plus de la secrétaire déjà en place à temps partiel. Les éditeurs - Springer-Verlag - ont généreusement accepté d'assumer la moitié du salaire des deux secrétaires. D'autres frais sont entraînés par les traductions et par une subvention aux éditeurs, correspondant aux abonnements à prix spécial réduit, à l'intention des membres de l'Association. Les frais généraux pourraient être réduits si l'on demandait aux auteurs de fournir leurs propres traductions de résumés; une démarche en ce sens devra être- faite.

Les frais du Secrétariat et de Diabetologia y compris, le Trésorier estime qu'un budget annuel de 33000.- fr. suisses doit être prévu pour 1966/67.

Le Dr. PFEIFFER poursuit en citant quelques propositions qui ont été faites pour réduie les dépenses de l'As. 
sociation. Il a été suggéré d'introduire une finance d'inscription pour les réunions annuelles; celle-ci donnerait droit aussi bien au volume de résumés qu'à certains repas. Il serait utile des continuer d'encourager la mise sur pied d'une exposition en relation avec les réunions annuelles.

Le Trésorier prie tous les membres de rechercher de nouveaux membres bienfaiteurs.

Quant au paiement des cotisations, le Dr. PFELFFer espère que les associations nationales voudront bien réunir ces petites sommes et nous les transférer en bloc, bien que l'adhésion à l'Association Européenne reste une affaire individuelle et que les membres des Associations nation. ales ne désireront pas forcément tous faire partie de l'Association Européenne.

Le Dr. Pfeiffer déclare qu'il s'est donné pour but d'obtenir 50000. - fr. suisses de cotisations annuelles. Une grande partie du travail en relation avec Diabetologia a été faite jusqu'ici bénévolement, mais cette situation ne peut se prolonger indéfiniment. En outre, un des buts de l'Association doit être de donner à de jeunes chercheurs un appui financier qui leur permette de participer aux réunions et d'y présenter des communications.

Le Trésorier conclut en informant l'assemblée qu'en l'absence du Dr. Coppo - un des vérificateurs de comptes - c'est le Dr. J. Mrrouze qui a contrôlé les livres avec l'autre vérificateur, le Dr. P. RAMBERT.

Les Drs. P. Rambert et J. Mrrouze sont confirmés dans leurs fonctions de vérificateurs de comptes, par acclamation.

\section{Rapport des vérificateurs de comptes}

Le Président invite le Dr. RAMBERT à présenter son rapport.

Le Dr. Raybert, en son nom et au nom du Dr. MrRouze, informe l'Assemblée que les comptes ont été excellement tenus et demande aux membres présents de donner décharge au Trésorier pour les comptes au 31 XII 65, ce qui est accepté à l'unanimité.

Le Président, au nom de l'Association, remercie les Drs. Rambert et Mirouze de leur collaboration et le Trésorier pour son concours compétent, la clarté de son rapport, et tout le travail qu'il accomplit.

\section{Rapport du secrétaire}

Le Président invite le Dr. Renold à présenter son rapport.

Le Secrétaire annonce d'abord que l'Association compte 905 membres actifs, parmi lesquels 500 ont payé leur cotisation annuelle. Des rappels seront envoyés en temps voulu à ceux qui ne s'en seraient pas encore acquittés. De ces 905 membres, 255 seulement sont abonnés à Diabetologia; il est recommandé à tous les membres de soutenir la revue.

Le Dr. Renold annonce ensuite que le nom des membres du Comité de l'Association, celui des membres d'honneur et des membres bienfaiteurs, sera imprimé régulièrement dans Diabetologia.

Abordant la réunion annuelle de l'Association, le Secrétaire souligne l'aide reçue d'un certain nombre de maisons. Il est sûr que chacun a apprécié le fait que les 180 communications dont les résumés ont été imprimés n'aient pas toutes été inscrites au programme; il demande l'avis des membres sur le rythme de présentation adopté cette année: 15 minutes suivies de 10 minutes de discussion, ce qui paraît préférable à des séances parallèles. En outre, les interventions de 2 minutes avec un maximum de 2 clichés semblent avoir satisfait chacun. A main levée, la majorité des membres présents se déclarent en faveur de la forme de réunion adoptée cette année.

Pour terminer, le Secrétaire rappelle que l'article 16 des Statuts prévoit que les membres du Comité de Direction doivent se retirer après 3 ans. Toutefois, pour éviter que le premier Comité exécutif (Président, 2 vice-prési- dents, Trésorier et Secrétaire) ne se retire en bloc, le Comité propose qu'il ne soit pas tenu compte, provisoirement, de l'article 16, de manière à permettre à ces membres de se retirer à tour de rôle, sur une période maximum de 5 ans, à partir de 1965 .

L'Assemblée générale approuve cette proposition et laisse le soin au Comité de Direction de décider de l'ordre de démission.

Le rapport du Secrétaire ne suscitant aucune question, le Président remercie très chaleureusement le Dr. $R_{\text {ENOLD }}$ de tout son travail, qui appelle toute la reconnaissance de l'Association.

\section{Rapport du rédacteur en chef de Diabetologia}

Le Président invite le Dr. OBERDISse, rédacteur en chef de Diabetologia, à présenter son rapport. Le Dr. OBERDISSE rappelle que le premier numéro de la revue est sorti en août 1965. Grâce à l'intense propagande faite par Springer-Verlag, les abonnés sont déjà presque 1000.

Les manuserits ont afflué de façon constante, mais leur qualité étant variable, certains ont dû être refusés. L'emploi de 3 langues a causé quelques difficultés majeures. Le but du Comité de Rédaction étant d'assurer une pa. rution régulière, il espère qu'à l'avenir tous les auteurs voudront bien envoyer leurs manuscrits en 3 exemplaires, pour éviter des photocopies; de plus, les résumés des articles devront être envoyés dans les 3 langues officielles. Le Comité de Rédaction est disposé à revoir les traductions mais ne peut assurer le travail de traduction lui-même.

La standardisation des abréviations est souhaitable; dans ce but, un comité restreint a été formé.

Deux membres du Conseil de Rédaction sont décédés: le Dr. G. Mohnike et le Dr. R.F. Ogiturie. Pour les remplacer, les Drs. G. Peteres (Lausanne) et G. Seifert (Hamburg) sont nommés et les Drs. T. SteEN-Olsen (Aarhus) et A. Hassemblat'T (Göttingen) viennent compléter le Comité.

Le Dr. Oberdisse souligne que les auteurs sont responsables de la lecture des épreuves et demande qu'ils s'en acquittent avec plus de soin pour réduire le travail du Comité de Rédaction, qui est responsable de la lecture de la mise en page finale.

Enfin, le Rédacteur en chef remercie les membres du Conseil de Rédaction pour leur aide et leurs conseils, ainsi que les membres du Comité de I'Association. Il remercie aussi le Dr. D.A.B. Young, le Dr. Loubarmînes, le Dr. DAwEK et le Dr. LIEBERMEISTER de leur concours de traducteurs.

Aucune question n'étant posée, le Président remercie le Dr. OBERDISSE de tout ce qu'il a fait pour le lancement et le développement de la revue et le prie de transmettre les remerciements de l'Association à ses collaborateurs.

\section{Désignation et élection de nouveaux membres} du Comité de direction

Le Président rappelle que, lors de la première assemblée générale, le Comité de Direction avait été chargé de décider d'un ordre de rotation pour les membres. Celui-ci a été tiré au sort; les membres qui se retirent en 1966 sont les Drs. Alrvisatos, Bartielmeimer, Chatn, Dérot et Ponpen. En 1967, ce sera le tour des Drs. JERSILD, LUFT, Maske, Rodriguez-Minon et Srgnorelit.

Le Président remercie les membres sortants de leur concours.

Lors de la 1ère Assemblée générale, 3 membres - les Drs. P.A. Bastente, T.R. Fraser et A. Loubatières avaient été élus au Comité de Direction pour la période de 1966 à 1969 . Il y avait done 2 places vacantes et le Comité de Direction propose les Drs. A. Czyzyr (Varsovie) et I. Paver (Bucarest) pour les occuper. Comme il n'y a pas d'autres propositions, les Drs. CzYzYK et PAveL sont élus par acclamation. 


\section{Prochaine séance}

Le Président annonce que la 3ème Assemblée générale aura lieu à Stockholm, Suède, au cours du 6ème Congrès de 1'International Diabetes Federation, qui se tiendra du 30 VII au 4 VIII 1967; l'heure et le lieu seront annoncés ultérieurement.

\section{Divers}

a) Liste des membres: Répondant à une question, le

Secrétaire annonce qu'il a l'intention de faire imprimer la liste des membres de l'Association au cours de l'année à venir.

Aucun autre sujet n'étant mis en discussion, la séance est levée.

Dr. F.G. Young

Président
Dr. A.E. RENOLD

Secrétaire

\section{Protokoll-Vorschlag der 2. Generalversammlung der Europäischen Gesellschaft für Diabetologie, abgehalten am Donnerstag, dem 7. Juli 1966 um 18.10 Uhr im Anatomischen Institut der Universität Aarhus, Dänemark}

\author{
Anwesend: Dr. F.G. Young (Präsident) \\ Dr. K. LUNDBAEK (Vizepräsident) \\ Dr. E. MARTIN (Vizepräsident) \\ Dr. E.F. PFeIfFer (Schatzmeister) \\ Dr. A.E. RENOLD (Sekretär) \\ und 108 Mitglieder der Gesellschaft
}

\section{Protokoll}

Der Vorstand berichtete, daß das Protokoll der Ersten Generalversammlung in der Juni-Ausgabe der Diabetologia $(2,1,72-74)$ des Jahres 1966 veröffentlicht wurde. Hierzu sind zwei Berichtigungen notwendig:

a) Punkt 3: Sowohl in der englischen als auch in der französischen Fassung des Protokolls war die Zahl der Mitglieder des Redaktionsausschusses versehentlich mit 17 anstatt 26 oder mehr angegeben worden.

b) Punkt 7: Lodiglich in der englischen Fassung sind die Namen der gewählten Kassenprüfer übergangen worden.

Nach Richtigstellung dieser Punkte wurde das Protokoll der ersten Generalversammlung angenommen, bestätigt, und vom Präsidenten unterzeichnet.

\section{Bericht des Präsidenten}

Bei der Eröffnung der Sitzung teilte der Präsident zunächst mit, daß er Dr. LuNDBaEK und seinen Kollegen den Dank der Gesellschaft für die Organisation der Tagung ausgedrückt habe. Die Erwartungen der Gesellschaft seien erfüllt worden. Der Präsident erwähnte, daß durch die Zusammenfassungen, die in zwei Sprachen vorlagen und durch die Großzügigkeit der Novo-Laboratorien zur Verfügung gestellt werden konnten, der Ablauf der Tagung und die zwanglose Diskussion erleichtert wurden.

Der Präsident sprach den Vorstandsmitgliedern den Dank der Gesellschaft für ihren aufopfernden Dienst aus und sagte, daß sich die Statuten in der Praxis bewährt hätten, obwohl der Sekretär zur Vermeidung eines gleichzeitigen Rücktritts sämtlicher Mitglieder des Vorstandes eine zeitweilige Aufhebung von Artikel 3, Abschnitt 16 vorschlagen müsse.

Der Präsident gab bekannt, daß der Verleihungsmodus für den Minkowski-Preis vom Beirat angenommen worden war. Die Qualifikation der Vorgeschlagenen sei sehr hoch gewesen. Er sei daher sicher, daß die Gesellschaft sich auf eine lange Reihe ausgezeichneter MinkowskiVorträge - wie schon vom ersten Preisträger, Dr. P.J. RANDIE, - freuen könne.

Das Preiskomitee bestehe aus vier vom Beirat ernannten Mitgliedern, von denen jährlich einer ausscheide, und einem Mitglied, das der Förderer, die Farbwerke Hoechst AG, vorschlägt. Dr. H. MAskig sei ausgeschieden; der Beirat habe an seiner Stelle Dr. A. LovBatxìdes ernannt. Dr. VON WASIEIEWSIKI sei von den Farbwerken Hoechst AG nominiert und in das Komitee aufgenommen worden.
Der Beirat war sich einig, daß in den Jahren, in denen die Internationale Diabetes Föderation in Europa taot, kein wissenschaftliches Treffen der Europäischen Geselischaft stattfinden sollte. Mit der Föderation wurde jedoch vereinbart, während des Kongresses der Föderation den Minkowski-Preis zu verleiben und auch den MinkowskiVortrag zu halten.

Der Vorsitzende gab weiterhin bekannt, daß der Beirat sich in dem Bestreben einig war, in Verbindung mit der Tagung der Gesellschaft eine Ausstellung abzuhalten; in Zukunft sollten die Tagungen der Gesellschaft vorzugsweise in kleinen Universitätsstädten stattfinden. Es war vorgeschlagen worden, für zukünftige Tagungen ein bestimmtes Datum für jedes Jahr festzusetzen; der Präsident bat diesbezüglich um Meinungsäußerung. Durch Er. heben der Hand sprach sich eine große Mehrheit der Generalversammlung für die jährliche Abhaltung der Tagung in der ersten Julihälfte aus, sofern äußere Umstände nicht dagegen sprächen.

\section{Bericht des Schatzmeisters}

Der Vorsitzende bat Dr. E.F. PFEIFFER um soinen Bericht. Der Schatzmeister begann mit der Aufstellung der Finanzlage der Gesellschaft:

Einnahmen 13.10.64 - 30.6.66: S.fr. 56597.95.

Ausgaben 13.10.64 - 30.6.66: S.fr. 19639.95

Bilanz

$$
\text { 30. 6. 66: } \overline{\text { S.fr. } 36958.00}
$$

Der größte Teil der Einnahmen stammte von 12 unterstützenden Mitgliedern der Gesellschaft; erfreulicherweise Konnte mitgeteilt werden, daß während der jetzigen Tagung zwei weitere unterstützende Mitglieder gewonnen wurden.

Bei Besprechung der Ausgaben hob Dr. E. F. PfeIfterR die ausgezeichnete Arbeit des Sekretariats in Genf hervor, dessen Kosten bemerkenswert niedrig gehalten worden seien; mit dem Anwachsen der Gesellschaft würden jedoch auch diese Ausgaben steigen; er schätze die jährlichen Kosten des Sekretariats für die 2 nächsten Jahre auf S.fr. 15000.-.

Die sonstigen Hauptausgaben verursache die Zeitschrift Diabetologia; der Schatzmeister gab bekannt, daß es nun unumgänglich geworden sei, für die mit dieser Publikation verbundene beträchtliche Arbeit eine weitere hauptamtliche Sekretärin einzustellen, Der Verleger (Springer-Verlag) erklärte sich großzügigerweise bereit, die Hälfte der Kosten beider Sekretärinnen zu übernehmen; Zablungen waren auch für Übersetzungsarbeiten notwendig, sowie für die Subvention, die dem Verlag für die Mitglieder der Gesellschaft bezahlt wurde, die die Zeitschrift Diabetologia zu einem ermäßigten Preis erhielten. Die Herstellungskosten könnten erniedrigt werden, wenn die Autoren die Zusammenfassungen ihrer Arbeiten in Zukunft selbst übersetzen würden. 
Für das Sekretariat und die mit der Publikation von Diabetologia verbundenen Kosten veranschlagte der Schatzmeister für $1966 / 67$ ein jährliches Budget von S.fr. $33000 .-$.

Dr. E. F. PfertFer ging anschließend auf einige Vorschläge zur Verminderung der Ausgaben der Gesellschaft ein. So war vorgeschlagen worden, bei zukünftigen Tagungen eine Teilnehmergebühr zu erheben, womit sowohl die Kosten des Druckes der Zusammenfassungen als auch von Veranstaltungen (Essen) abgegolten werden könnten. Es sei auch beabsichtigt, in Verbindung mit den Tagungen eine Ausstellung abzuhalten.

Der Schatzmeister bat sämtliche Mitglieder, alles zu tun, um mehr unterstützende Mitglieder zu gewinnen.

Dr. Pfeiffer drückte die Hoffnung aus, daß in $\mathrm{Zu}$ kunft bei der Ũberweisung der Mitgliedsbeiträge die nationalen Gesellschaften mitwirken würden, obwohl die Mitgliedschaft in der Europäischen Gesellschaft eine persönliche Angelegenheit sei, und nicht sämtliche Mitglieder nationaler Gesellschaften Mitglieder der Europäischen Gesellschaft werden wollten.

Dr. Premfrer stellte fest, daß sein Ziel die jährliche Beschaffung von S.fr. 50000.- sei. Der größte Teil der Redaktionsarbeit für Diabetologia würde gegenwärtig ohne Entgelt durchgeführt, was auf die Dauer nicht verlangt werden könne. Daneben hoffe er, daß die Gesellschaft in der Lage sein werde, jungen Wissenschaftlern eine finanzielle Unterstïtzung zu geben, die es ihnen ermögliche, an Tagungen teilzunehmen and Arbeiten vorzutragen.

Der Schatzmeister schloß seinen Bericht und stellte fest, daß in Abwesenheit von. Dr. Coppo, als einer der beiden Kassenprüfer, Dr. MrRouze gebeten wurde, seinen Platz einzunehmen. Dr. P. Ramert und Dr. Mirouze wurden für $1966 / 67$ als Kassenprüfer vorgeschlagen und bestätigt.

In Beantwortung einer Anfrage stellt der Schatzmeister noch fest, daß etwa S.fr. 6300. - aus Mitgliedsbeiträgen eingegangen seien.

\section{Bericht der Kassenprüfer}

Dr. RAMBERT teilte der Generalversammlung auch im Namen von Dr. J. Mrrouze mit, daß die Buchführung in Ordnung war und bat die Generalversammlung, den Schatzmeister für die Zeit bis zum $31.12 .65 \mathrm{zu}$ entlasten. Die Generalversammlung nahm den Vorschlag einstimmig an.

Der Präsident dankte den Herren Dr. RAMBERT und Dr. Mirouze im Namen der Gesellschaft für ihre Arbeit; auch dem Schatzmeister sprach er für seinen guten und klaren Bericht und für seine schwere Arbeit den Dank der Gesellschaft aus.

\section{Bericht des Sekretärs}

Der Präsident bat Dr. A.E. Renold um seinen Bericht.

Der Sekretär sagte zu Beginn, daß von den 905 aktiven Mitgliedern der Gesellschaft ungefähr 500 ihre Mitgliedsbeiträge bezahlt hätten. Demnächst würden Mahnungen an die Säumigen gesandt werden. Von den 905 Mitgliedern hätten nur 255 die Zeitschrift Diabetologia abonniert; alle Mitglieder wurden aufgerufen, diese Zeitschrift zu unterstützen.

Dr. Renotn kündigte dann an, daß die Namen der Mitglieder des Beirates, der Ehren-Mitglieder und der unterstützenden Mitglieder in den kommenden Ausgaben von Diabetologia abgedruckt würden.

Auf die Gestaltung der Tagungen eingehend, hob der Sekretär hervor, das es ohne die großziugige Unterstützung einiger Firmen nicht möglich gewesen wäre, die Zusammenfassungen rechtzeitig zur Verfügung zu stellen.
Er bat die Mitglieder um Meinungsäußerung über den $A b$ lauf der Tagung in Aarhus, bei der die Vorträge auf 15 Minuten und die anschließenden Diskussionen auf $10 \mathrm{Mi}$. nuten begrenzt wurden. Dieser Form der Kongreßgestaltung wurde im allgemeinen der Vorzug gegeben. Daneben bat sich die Diskussionsbemerkung (2 Minuten, 2 Dias) bewährt.

Die MehrzahI der Mitglieder stimmte durch Erheben der Hand für eine Fortsetzung dieses Modus bei zulkünftigen Tagungen.

Schließlich berichtete der Sekrretär, daß Artikel 5, Abschnitt 16 der Satzung die Ausscheidung aller Mitglieder des Beirats nach 3 Dienstjahren vorsehe. Da aber das gleichzeitige Ausseheiden des ersten Vorstandes allgemein als nachteilig empfunden wird, schlug der Beirat vor, diesen Abschnitt der Satzung vorübergehend außer Kraft zu setzen und für das Ausscheiden des ersten Vor standes eine Zeitspanne von höchstens 5 Jahren (ab 1965) vorzusehen. Die Generalversammlung nahm diesen Vorschlag an und überließ es dem Beirat, die Reihenfolge des Ausscheidens festzusetzen.

Zum Bericht des Sekretärs gab es keine Wortmeldungen, der Präsident dankte daher im Namen der Gesellschaft Dr. A.E. Renold für seine Arbeit.

\section{Bericht des Schriftleiters von Diabetologia}

Der Präsident bat Dr. K. OBERDIsse, Schriftleiter von Diabetologia, um seinen Bericht. Dr. OBERDISse teilte mit, daß die erste Nummer von Diabetologia im August 1965 erschienen sei. Dank der hohen Qualität der eingesandten Arbeiten und dank der intensiven Werbung durch den Verleger (Springer-Verlag) betrage die derzeitige Abonnentenzahl annähernd 1000.

Es gingen ständig Manuskripte ein, deren Qualität jedoch unterschiedlich sei, so daß einige abgewiesen werden mußten. Große Schwierigkeiten wurden durch die Aufnahme von Arbeiten in drei Sprachen verursacht; trotzdem ist es weiterhin das Ziel der Herausgeber, das regel. mäßige Erscheinen der Zeitschrift sicherzustellen. Es sei zu hoffen, daß in Zukunft sämtliche Autoren ihre Manuskripte in 3-facher Ausfertigung einsenden, um das sonst notwendige Vervielfältigen einzusparen. Außerdem sollten die Zusammenfassungen der Arbeiten in sämtlichen Sprachen eingesandt werden. Die Herausgeber sind gerne bereit, bei der Korrektur der Übersetzungen behilflich zu sein, es ist ihnen jedoch unmöglich, die ganze Übersetzungsarbeit durchzuführen.

In dem Bestreben, die Abkürzungen einheitlich zu gestalten, wurde ein Ausschuß gebildet, der entsprechende Empfehlungen ausarbeiten soll.

Zwei Mitglieder des wissenschaftlichen Beirates, Dr. G. MohNIKE und Dr. R.F. OGIIVIE, sind gestorben. Als Nachfolger wurden Dr. G. Peters (Lausanne) und Dr. G. SeIFERT (Hamburg) vorgeschlagen; außerdem wurden Dr. T. Steme-Olsen (Aarhus) und Dr. A. Hasselarat' (Göttingen) in den Beirat gewählt.

Dr. OBerdrsse wies darauf hin, da. die Autoren selbst für die Durchsicht der Korrekturfahnen zu sorgen hätten, und bat darum, diese Arbeit sorgfältiger auszufïhren, um die Herausgeber, die für die Überprüfung der Umbrucharbeiten verantwortlich seien, zu entlasten.

Zum Schluß sprach Dr. OBERDIsse den Herausgebern und den Mitgliedern des wissenschaftlichen Beirates für ihre Unterstützung seine Anerkennung aus, und dankte auch den Herren Dr. D.A.B. Young, Dr. Loubatrìkes, Dr. Daweike und Dr. LteberRmeister für ihre Hilfe bei den Übersetzungsarbeiten.

$\mathrm{Zu}$ diesem Thema gab es keine Wortmeldungen. Der Präsident dankte daraufhin Herrn Dr. OBERDIsse für seinen Beitrag zum erfolgreichen Start der Zeitschrift und bat ihn, auch seinen Kollegen den Dank der Gesellschaft weiterzuleiten. 
7. Nominierung und Wahl newer Mitglieder des Beirats

Der Präsident erinnerte die Generalversammlung daran, daß auf der 1. 'Tagung dem Beirat die Verantwortung auferlegt worden sei, die Reihenfolge des Ausscheidens der Mitglieder des Beirats zu regeln. Das geschah durch Losentscheid. Die ausscheidenden Mitglieder für 1966 waren Dr. Alivisatos, Dr. BartelhetMer, Dr. CHAin, Dr. DÉRot und Dr. PoMpen; für 1967 werden es Dr. Jersird, Dr. LufT, Dr. Maske, Dr. RodriguezMinon und Dr. SignoRELLI sein.

Der Präsident dankte den ausscheidenden Mitgliedern für ihre Hilfe. Auf der ersten Generalversammlung waren für 1966 - 1969 drei Mitglieder, nämlich Dr. P.A. BAsTENIE, Dr. T.R. Fraser und Dr. A. Loubattìres, in den Beirat neu gewählt worden. Zwei Plätze waren somit noch unbesetzt, für die der Beirat die Herren Dr. A. CzyzyK (Warschau) und Dr. I. PAVEL (Bukarest) vorschlug. Sonst gab es keine Nominierungen. Dr. Czyzyk und Dr. I. Paveı wurden durch Akklamation gewählt

\section{Nächste Versammlung}

Der Präsident kündigte an, daß die dritte General versammlung während des 6 . Kongresses der Internatio. nalen Diabetes Föderation, der vom 30. Juli bis 4 . August 1967 in Stockholm, Sehweden, stattfindet, abgehalten werden soll; Zeitpunkt und Ort der Versammlung werden noch bekanntgegeben.

\section{Sonstiges}

a) Mitglieder-Liste: In Beantwortung einer Frage sagte der Sekretär, daß das Sekretariat die Absicht habe, im nächsten Jahr eine gedruckte Liste der Mitglieder der Gesellschaft herauszugeben.

Die Sitzung wurde geschlossen.

Dr. F.G. Young

Präsident
Dr. A. E. Renold Sekretär

\section{Université de Paris, Faculté de Médecine, Chaire de Clinique Médico-Socjale du Diabète et des Maladies} de la Nutrition

\section{Journées Annuelles de Diabétologie}

Les 8èmes Journées de Diabétologie de l'Hôtel-Dieu, auront lieu les 18, 19 et 20 mai 1967, sous la présidence des professeurs M. DeRot et H. Bour, avec la collaboration de diabétologues français et étrangers.
Les principaux sujets exposés seront: le Glucagon, le Diabète de l'Enfant et sa Prévention, l'Oeil Diabétique.

Pour tous renseignements, s'adresser au Docteur M. Rathery, secrétaire des Journées de Diabétologie, HôtelDieu de Paris, 1 Place du Parvis Notre Dame, Paris $\left(4^{\circ}\right)-$ $32607-79$.
Die Deutsche Diabetes-Gesellschaft - Ausschuß Pädiatrie veranstaltet vom 26. bis 27. Mai 1967 in Bonn unter der Leitung von Prof. Dr. HUNGERLAND eine Arbeitstagung mit dem Thema:

$$
\text { „Die Betreuung des diabetischen Kindes" }
$$

Anmeldung: Priv.-Doz. Dr. DuEmer, Univ.-Kinderklinik, Bonn, Koblenzer Str. 119

\section{Vorläufiges Programm:}

Die Kost des diabetischen Kindes

Biguanide und Sulfonylharnstoffe in der Behandlung und Diagnostik des kindlichen Diabetes
Insuline und Insulinbehandlung

Ambulante Betreuung, Einstellung und Überwachung des diabetischen Kindes

Diabetes und Schule

Schulung und Fortbildung der Eltern

Die Durchführung von Ferienlagern für diabetische Kinder

Dauerheime für diabetische Kinder und Jugendliche

Die Ergebnisse der pädiatrischen Behandlung aus der Sicht des Internisten. 